\title{
Social Cognition, Behaviour and Relationship Continuity in Dementia of the Alzheimer Type
}

\author{
Blanca Poveda, ${ }^{1}$ Katherine Osborne-Crowley, ${ }^{2}$ Kenneth Laidlaw, ${ }^{3}$ Fiona Macleod ${ }^{4}$ \\ and Kevin Power ${ }^{5}$ \\ ${ }^{1}$ NHS Lothian, Clinical Neuropsychology Department, Astley Ainslie Hospital, Edinburgh, UK \\ ${ }^{2}$ School of Psychology, University of South Wales, Syndney, Australia \\ ${ }^{3}$ Norwich Medical School, Faculty of Medicine and Health Sciences, University of East Anglia, Norwich \\ Research Park, Norwich, UK \\ ${ }^{4}$ NHS Tayside Older People, Psychological Therapies Services, Stracathro Hospital, Brechin, UK \\ ${ }^{5}$ University of Stirling, University of Stirling, Stirling, UK
}

\begin{abstract}
Social cognition can be impaired in a range of neuro-degenerative conditions, yet the impact of these difficulties on behaviour and social relationships is not yet fully understood. This study assessed social cognition in 27 participants with Dementia of the Alzheimer Type (DAT) and their co-residing partners $(N=27)$ and explored the relationships between social cognition, cognitive ability, relationship continuity and behaviour following diagnosis. In line with previous research, participants with dementia scored lower on social cognition tasks compared to their partners. Behaviour changes such as apathy, disinhibition and agitation in participants with dementia were significantly related to relationship continuity; however, no significant associations were found with measures of social cognition. The results of this study are discussed within a therapeutic context and in line with current guidelines and policies.
\end{abstract}

Keywords: social cognition, Alzheimer's disease, dementia, theory of mind, emotion recognition

\section{Introduction}

The most commonly diagnosed dementia is Dementia of the Alzheimer's Type (DAT) (McKhann et al., 1984). Individuals with a diagnosis of probable DAT typically present with primarily cognitive symptoms such as difficulties with memory, language or visual and spatial functions (McKhann et al., 1984). However, since DAT does entail frontal atrophy, particularly in the later stages of the disease, individuals may also experience difficulties in attention, executive functioning, social cognition and behaviour (McKhann et al., 1984), including agitation, apathy, mood changes, hallucinations or delusions (Jalbert, Daiello, \& Lapane, 2008). These changes in social and emotional behaviour after dementia are a major risk factor for increased carer burden (Burke \& Morgenlander, 1999; Coen et al., 1997; Cohen et al., 1993;
Hebert, Dubois, Wolfson, Chambers, \& Cohen, 2001; Hsieh, Irish, Daveson, Hodges, \& Piguet, 2013) and are often reported to be more distressing and disabling than cognitive changes (Steele et al., 1990).

Importantly, carers for people with dementia are often intimate partners, who may have difficulty adjusting to significant changes in the relationship (Garand et al., 2007). One important variable related to well-being of the carer is their perceptions of the continuity/discontinuity of the relationship. Perceived discontinuity refers to perceptions of the relationship as being essentially changed and radically different as a result of dementia, rather than as a continuation of the pre-morbid relationship. Although it is well-established that behavioural changes after dementia are related to carer burden, it is not yet 
known whether these behavioural changes are associated with perceived discontinuity of the relationship. This relationship might be expected since behavioural changes such as disinhibition, aggression and apathy fundamentally affect the way in which a person interacts with others, which is the cornerstone of interpersonal relationships. The current study was the first to investigate this proposed relationship between behavioural change and discontinuity of relationship.

The second aim of this study was to determine whether social cognition deficits underlie these disabling changes in behaviour after DAT. An individual's ability to respond appropriately in a social situation relies on their skilful integration of semantic, syntactic, contextual and paralinguistic information as well as on their pragmatic knowledge and ability to take visual perspectives, understand emotions and utilise theory of mind (ToM) (ShanyUr et al., 2012). Impairments in these critical social cognitive skills may lead to behaviours such as disinhibition, aggression or even apathy. For example, if someone struggles to perceive another person's emotions or understand negative social feedback, they may say things that could be perceived as offensive or they may not react to other's feelings. Moreover, difficulties understanding sarcasm or jokes, could lead to defensiveness or aggressive behaviour.

Research into the role of social cognition in behaviour change in neuro-degenerative conditions is still within its infancy, with the majority of work conducted on Behavioural variant of FrontoTemporal Dementia (bvFTD). Patients with bvFTD not only experience changes in behaviour, personality and motivation (Rascovsky, Hodges, \& Knopman, 2011), but also have severe deficits in emotion processing and emotion recognition (Kipps, Nestor, Acosta-Cabronero, Arnold, \& Hodges, 2009b; Kumfor et al., 2015; Lavenu, Pasquier, Lebert, Petit, \& Van der Linden, 1999). Keane, Calder, Hodges, \& Young (2002) found that five of their individuals with bvFTD were significantly impaired in recognising emotions and suggested that impairments in emotion processing might underpin reported changes in social behaviour. In line with this, Kipps and colleagues (2009a) reported significant correlations between mood sub scores, performance on an emotion recognition task and the Cambridge Behaviour Inventory (Bozeat, Gregory, Ralph, \& Hodges, 2000). Similarly, Shany-Ur et al. (2012) reported a link between social cognition as assessed on the The Awareness and Social Inference Test (TASIT) (McDonald, Flanagan, Rollins, Kinch, 2003) and informants' ratings of empathy, perspective taking and neuro-psychiatric symptoms in a bvFTD sample.
Furthermore, Gregory et al. (2002) found a significant relation between performance on tests of ToM (an aspect of social cognition) and neuropsychiatric and behavioural dysfunction on the Neuropsychiatric Inventory (NPI) in individuals with bvFTD. Thus, in the bvFTD literature, the evidence suggests that social cognition plays an important role in neuro-behavioural outcome.

However, there is a very little research examining the relationship between social cognition and behaviour in DAT. This paucity of research may reflect the view that people with DAT do not suffer from problems of social cognition. There is, however, increasing evidence that individuals diagnosed with DAT do suffer from impairments in social cognition, specifically emotion processing (Phillips, Scott, Henry, Mowat, \& Bell, 2010), including recognising emotions from faces (Hargrave, Maddock, \& Stone, 2002), voices (Roberts, Ingram, Lamar, \& Green, 1996) and body movements (Koff, Zaitchik, Montepare, \& Albert, 1999). Only one study to date has investigated the relationship between social cognition and behaviour in DAT. Shimokawa et al. (2001) explored the relationship between the Emotion Recognition Test (ERT) (Shimokawa et al., 2001), and interpersonal behaviour changes as assessed by the Interpersonal Behaviour Checklist (IBC, Shimokawa et al., 2001). They found that behaviour change on the IBC scale correlated with ERT scores but not with Mini-Mental State Examination (MMSE) performance, suggesting that the behaviour of patients with DAT does not depend on deterioration of cognitive ability but rather on a decreased ability for emotion comprehension (Shimokawa et al., 2001).

Although this study does attest to the relationship between social cognition and behaviour in DAT, a major drawback is the use of tests of social cognition that do not have ecological validity. Studies exploring emotion recognition in individuals with DAT have largely relied on Ekman and Friesen's (1976) 60 Faces Test (FEEST; Henry et al., 2008), a static display of black and white photographs of human faces displaying one of the six basic emotions: 'happy', 'disgust', 'fear', 'sadness', 'anger' and 'surprise', as well as neutral faces displaying no emotion. Although results from these studies suggest impairments across all stages of the disease progression (e.g., Hargrave, Maddock, \& Stone, 2002; Phillips et al., 2010), these tasks are highly artificial and therefore limited in the extent to which they reflect real-life social exchanges (McDonald et al., 2003). Indeed, it has been demonstrated that people with DAT perform much better when asked to identify emotions from realistic, audiovisual displays compared to static 
black and white photos (Henry and colleagues, 2008). Henry and colleagues (2008) concluded that it is possible that more traditional measures of affect recognition, i.e., FEEST, over-estimate the degree of impairment that participants with DAT experience in their day-to-day life. Thus, the current study aimed to examine the relationship between social cognition and behaviour using an ecologically valid measure of emotion recognition.

Finally, the current study also aimed to determine whether social cognition impairments observed in people with DAT can be accounted for by their non-social cognitive deficits. Real-time social exchanges demand attention, language, information processing skills and memory. It is quite possible that these factors play an important role in any social impairments experienced by people with DAT. Thus, it was predicted that general cognitive ability will account for a significant amount of the variance in relation to social cognition in participants with DAT.

Thus, the current study examined the relationships between general cognitive ability, social cognition, problem behaviours and relationship continuity. The main hypotheses for this study were (i) participants with DAT would be impaired on a social cognition task in comparison to their partners and that this would be partially accounted for by general cognitive ability and (ii) there would be a significant negative correlation between the, i.e., social cognition task and behaviour ratings in participants with DAT, particularly with regard to behaviours such as apathy, disinhibition, irritability and agitation and (iii) that social cognition and behaviour would be related to relationship continuity as reported by partners.

\section{Method}

\section{Participants}

Twenty-seven participants with DAT and their partners were recruited. All couples were currently living together and had been married between 22 and 73 years $(M=51.81, S D=10.83)$. Of the participants with dementia, 17 were female and 10 were male; age ranged from 71 to 94 years $(M=$ $78.9, S D=4.83)$. Partners were aged between 65 and 96 years $(M=78, S D=6.22)$. Consultant old age psychiatrists within the relevant health boards had made all dementia diagnoses, between 1 and 8 years previously (time since diagnosis $M=3.12$, $S D=1.68)$.

Inclusion criteria for participants with DAT were (i) a medical diagnosis of probable DAT according to the National Institute of Neurological and Communicative Disorders and Stroke and the
Alzheimer's Disease and Related Disorders (McKhann et al., 2011) and DSM-V (APA, 2013) criteria, (ii) mild-to-moderate DAT with a score between 0.5 and 2 in the Clinical Dementia Rating (CDR) (Morris, 1997), (iii) an absence of major depression or psychiatric disorder, as defined by DSM-V criteria (APA, 2013), (iv) living with a partner who is willing to participate, (v) English spoken fluently, (vi) able to give informed consent to participate in the present research and (vii) living at home with their partner.

All participants were recruited through the Scottish Dementia Clinical Research Network (SDCRN) across two health boards in Scotland. The study received ethical approval from the East of Scotland Research Ethics Service (EoSRES-1) in May 2012 and was conducted in accordance with the Declaration of Helsinki and all volunteers gave their informed consent to participate.

\section{Measures}

General cognitive ability. The Addenbrooke Cognitive Examination Revised (ACE-R) (Mioshi et al., 2006) is a brief, sensitive and specific cognitive screening test that incorporates five subdomain scores: orientation/attention, memory, verbal fluency, language and visuospatial abilities. Mioshi et al. (2006) found sound psychometric properties for this measure, with good reliability $(\alpha=0.8)$ and validity, showing significant correlations with the CDR $(r=.321, p<.01)$. Four participants with DAT and two partners were excluded from the analysis as they were aged over 86 years.

Social cognition. TASIT (McDonald et al., 2003) is an audio-visual tool designed for the clinical assessment of social perception with alternate forms for re-testing. Part I, the ERT (TASITERT), shows 28 short video-vignettes (20-40 seconds each) of individuals depicting one of seven emotions: 'happy', 'surprised', 'neutral', 'sad', 'angry', 'fear' or 'disgusted'. Part II, the Social Inference-Minimal (TASIT-SIM) test, shows 15 short video-vignettes (20-40 second each) of everyday conversational exchanges. This test examines a person's understanding of conversational meanings determined by paralinguistic cues such as facial expression, tone of voice or gestures. The video vignettes in this test use neutral scripts, which are enacted by professional actors and can represent either 'sincere' or 'sarcastic' (simple and complex) social exchanges. An individual's ability to understand these social exchanges is then assessed using four questions regarding the actor's beliefs (i.e., what they know), meaning (i.e., what they mean by what is said), intentions (i.e., what 
they intend to do) and feelings (i.e., what they feel) (McDonald et al., 2003).

Part III, the Social Inference-Enriched test (TASIT-SIE), shows 16 short video-vignettes (2040 seconds each) of everyday conversational exchanges. Each vignette contains a literally untrue comment enacted in one of two ways: As sarcasm meant to amplify the truth or as a lie meant to conceal or minimise the truth. This test distinguishes between visual and text cues to determine the meaning of paralinguistic features and assesses an individual's understanding of the situation using the same four questions as in Part II: beliefs, meaning, intentions and feelings (McDonald et al., 2003). As per TASIT manual (McDonald, Flanagan, \& Rollins, 2002), composite scores can be created in all three parts of the TASIT by adding the relevant sub scores. The composite scores in each part are Part I include 'positive', (i.e., the sum of 'surprise', 'happy' and 'neutral' scores) 'negative' (i.e., the sum of 'revolted', 'sad', 'angry' and 'anxious' scores) and 'Total ERT' (i.e., the sum of both 'positive' and 'negative' total scores), and part II include 'sincere', 'simple sarcasm', 'complex sarcasm' and 'Total SIM' and part III include 'textual', 'visual' and 'Total SIE' composite scores.

Test-retest reliability for TASIT ranges from 0.74 to 0.88 . TASIT has significant associations with other measures of social cognition (e.g., FEEST, $r=.69, p<.01$ [TASIT-ERT] $r=.50$, $p<.01$ [TASIT-SIM] $r=.37, p<.01$ [TASITSIE]; First/second-order ToM $r=.68, p<.05$ [TASIT-SIM]) (McDonald et al., 2006).

Relationship continuity. The Birmingham Relationship Continuity Measure (BRCM) (Riley et al., 2013) has been validated to measure relationship continuity when caring for a partner with dementia. The BRCM is a 26-item instrument measuring carers' perceived continuity of spousal relationship, where one individual in the couple cares for the other, due to a diagnosis of dementia. The BRCM contains six domains: (i) changes in relationship; (ii) changes to the person; (iii) changes in feelings; (iv) sense of loss; (v) sharing and togetherness and (vi) expressions of affection and attachment. Each item in the BRCM is scored using a Likert scale from 1 'disagree a lot' to 5 'agree a lot'. The psychometric properties of this scale were assessed by Riley et al. (2013) and showed good internal consistency (Cronbach's $\alpha=.94$ ), good test-retest reliability $(\alpha=.96)$ and good concurrent validity (e.g., Closeness and conflict scale, $r=.43, p<.05$; (Marwit-Meuser Care giver Grief Inventory [Marwit \& Meuser, 2002] $r=.54, p<.01)$. Scores for the relationship continuity scale as rated by part- ners on this study were normally distributed ( $M=$ $76.04, S D=22.39$ ).

Neuro-behavioural problems. The NPI (Cummings et al., 1994; Kaufer et al., 1998) is an interview measure that assesses the presence of neuropsychiatric disturbances in a patient with dementia from the information provided by an informed care giver. A clinician conducts the interview with each of the carers enrolled in the study but not in the presence of the partner with dementia. The NPI assesses 12 common neuropsychiatric disturbances in dementia (delusions, hallucinations, agitation, dysphoria, anxiety, apathy, irritability, euphoria, disinhibition, aberrant motor behaviour, night-time behaviour disturbances, and appetite and eating abnormalities). For each domain, a screening (yes/no) question is initially given to determine whether problems in that domain are present. If the screening question determines that problems in that domain are present, the informant is asked to indicate the severity of behaviour within each domain, as measured on a three-point Likert scale, the frequency, as measured on a four-point scale, and the distress these behaviours cause is measured on a five-point scale. For each behavioural domain, there are four scores that can be calculated: a frequency, severity, total (frequency $\times$ severity) and a care giver distress score. Cummings (1997) established content validity (subjectively), concurrent validity (e.g., Hamilton Depression scale [Hamilton, 1960], all correlations $p<.05)$, inter-rater reliability $(93.6-100 \%)$ and test-retest reliability $(r=.79, p<.01$ [frequency], $r=.86, p<.01$ [severity]) of the NPI. A total NPI score can be calculated by adding the scores of the first 10 domain scores together. In most cases, the two neuro-vegetative items (appetite and night time behaviour disturbance) are not included in the NPI total score. The distress score is not included in the total NPI score. In this study, the total distress score is generated by adding together the scores of the first 10 items of the NPI distress questions.

Additional measures. Additionally, a pre-morbid measure of intelligence, the Wechsler Test of Adult Reading (WTAR) (The Psychological Corporation, 2001), was administered to all participants (see, Duff, Chelune \& Dennett, 2011). Finally, a screen for emotional distress, the Hospital Anxiety and Depression Scale (HADS) (Zigmond \& Snaith, 1983) was taken for all participants. 
TABLE 1

Results of Group Comparisons for Cognitive and Emotional Functioning Variables

\begin{tabular}{|c|c|c|c|c|c|c|c|c|c|}
\hline & \multicolumn{3}{|c|}{ DAT } & \multicolumn{3}{|c|}{ Partners } & \multirow[b]{2}{*}{ U-test } & \multirow[b]{2}{*}{$Z$} & \multirow[b]{2}{*}{ Sig. } \\
\hline & $N$ & Median & Range & $N$ & Median & Range & & & \\
\hline Age & 27 & 77.5 & $71-94$ & 27 & 78 & $65-96$ & 456.5 & 1.59 & n.s \\
\hline WTAR std. & 26 & 110 & $86-123$ & 25 & 112 & $92-125$ & 275.5 & 1.54 & n.s \\
\hline ACE-R & 23 & 70.50 & $41-83$ & 25 & 94 & $81-100$ & 13 & 6.10 & $p<.001$ \\
\hline Anxiety & 27 & 9 & $3-14$ & 27 & 4 & $1-12$ & 298.5 & -1.15 & n.s \\
\hline Depression & 27 & 4 & $0-8$ & 27 & 4 & $0-11$ & 354.5 & -0.18 & n.s \\
\hline NPI total & 27 & 8 & $0-60$ & & & & & & \\
\hline BRCM & & & & 27 & 81 & $23-108$ & & & \\
\hline
\end{tabular}

Note. N: number of participants, WTAR std: Wechsler Test of Adult Reading Standard Score, ACE-R: Addenbrooke's Cognitive Examination Revised, NPI: Neuropsychiatric Inventory; U-test: Mann-Whitney U-statistic, Z: standardised test statistic, Sign: significance level, n.s: not significant.

\section{Results}

\section{Exploratory Data Analysis}

The study contacted 132 couples, of which 27 agreed to participate $(20 \%)$. Data were initially examined for normality of the distribution. Tests of skewness and kurtosis were performed for the variables: age and total scores on the WTAR, ACE-R, BRCM, NPI-D, HADS-A, HADS-D and TASIT (ERT, SIM and SIE). A ceiling effect was identified for partners' ACE-R total scores. Partners' data were non-normally distributed for total scores on the ACE-R $(D[27]=.02, p<.05)$, HADS-A $(D[27]=.0, p<.05), \mathrm{NPI}(D[27]=.0$, $p<.05)$, TASIT-ERT $(D[27]=.02, p<.05)$ and TASIT-SIE $(D[27]=.0, p<.05)$. The participants with DAT group data were found non-normally distributed for TASIT-SIE $(D[22]=.04, p<$ $.05)$ and HADS-D $(D[27]=.02, p<.05)$. Data for the relevant variables were transformed using a $\log 10$ transformation in an attempt to correct for distributional difficulties. However, even after transformation, tests of normality still showed data for these variables as non-normally distributed. Levene's test was significant $(p<.01)$ for the ACE-R, $F(1,52)=16.70, p<.01$, TASIT-SIM, $F(1,551)=14.52, p<.01$ and TASIT-SIE, $F$ $(1,48)=6.37, p<.01$, indicating that variances were significantly different and the homogeneity of variance assumption was not tenable for these scores. As such, non-parametric testing (MannWhitney U-tests) was used to compare groups on WTAR, ACE-R scores and social cognition scores. Non-parametric measures of association (Spearman's Rho) were used to test associations between social cognition scores, relationship continuity, partners' mood, cognitive functioning skills and proxy ratings of behaviour. In order to account for
Type I error in multiple correlations, the critical $\alpha$ level was reduced to .01 as recommended by Field (2009). Not all participants with DAT were able to complete every part of the TASIT, part I $(N=26)$, part II $(N=23)$ and part III $(N=22)$. The study used missing value analysis as recommended by Field (2009) to manage missing data in our database, i.e., a numeric code was used to represent the missing values in the data. Only five participants were unable to complete TASIT-SIE, the most complex of the three TASIT tasks.

\section{Pre-morbid IQ and Cognitive and Emotional Functioning}

Descriptive statistics for age, standard WTAR score and ACE-R are displayed in Table 1. The two groups were matched for age and estimated premorbid cognitive ability. Significant differences, $\mathrm{U}(48)=13, Z=6.1, p=.0005$, were found between participants with DAT and their partners' general cognitive ability as measured by the ACER. No significant differences were found in anxiety or depression scores on the HADS between partners and participants with DAT.

\section{Social Cognition Results}

To compare performance between partners and participants with DAT on TASIT (Part 1: ERT, Part 2: SIM and Part 3: SIE subtest) scores, non-parametric Mann-Whitney U-tests were performed. Table 2 outlines the results of these group comparisons. On TASIT-ERT, there were significant differences between participants with DAT and their partners for all emotions with the exception of happy. Overall, there were significant differences between groups for positive, negative and 
TABLE 2

Performance on the TASIT for Participants with DAT and Their Partners

\begin{tabular}{|c|c|c|c|c|c|c|c|c|c|}
\hline & \multicolumn{3}{|c|}{ DAT dementia } & \multicolumn{3}{|c|}{ Partners } & \multirow[b]{2}{*}{ U-test } & \multirow[b]{2}{*}{$Z$} & \multirow[b]{2}{*}{$r$} \\
\hline & $N$ & Median & Range & $N$ & Median & Range & & & \\
\hline TASIT-ERT total & 26 & 15 & $7-26$ & 27 & 24 & $20-28$ & 20 & $5.91^{*}$ & -0.81 \\
\hline ERT negative emotions & 26 & 6.5 & $3-12$ & 27 & 11 & $8-12$ & 36 & $5.64^{*}$ & -0.77 \\
\hline ERT positive emotions & 26 & 9 & $3-14$ & 27 & 15 & $9-16$ & 29 & $5.77^{*}$ & -0.79 \\
\hline TASIT-SIM total & 23 & 38 & $11-47$ & 27 & 49 & $38-58$ & 29 & $5.49 *$ & -0.78 \\
\hline SIM sincere & 23 & 14 & $9-20$ & 27 & 18 & $14-20$ & 124 & $3.66^{*}$ & -0.52 \\
\hline SIM simple sarcasm & 23 & 10 & $2-20$ & 27 & 16 & $8-20$ & 123 & $3.66^{*}$ & -0.52 \\
\hline SIM complex sarcasm & 23 & 11 & $0-15$ & 27 & 17 & $7-20$ & 56.5 & $4.97^{*}$ & -0.71 \\
\hline TASIT-SIE total & 22 & 17.5 & $10-27$ & 27 & 12 & $8-15$ & 12 & 5.76 & -0.82 \\
\hline SIE lie & 22 & 10 & $4-14$ & 27 & 14 & $10-16$ & 44 & 5.12 & -0.73 \\
\hline SIE sarcasm & 22 & 10 & $2-20$ & 27 & 16 & $8-20$ & 31.5 & 5.38 & -0.77 \\
\hline
\end{tabular}

Note. N: number of participants, U-test: Mann-Whitney U-statistic, $r$ : effect size, $Z$ : standardised test statistic, ${ }^{*}$ : significant at $p<.001$ ERT: Emotion recognition test, SIM: Test of social inference minimal, SIE: Test of social inference enriched.

total TASIT-ERT, where partners obtained higher scores.

On TASIT-SIM, the participants with DAT had lower composite 'sincere', 'simple sarcastic' and 'complex sarcastic' scores, as well as lower overall scores compared to their partners. Similarly, on TASIT-SIE participants with DAT had lower composite 'lie' and 'sarcasm' and total scores compared to their partners.

Since the ACE-R scores were highly variable in the DAT group indicating a range of severity of DAT, a follow-up analysis was conducted in order to determine whether differences between groups on social cognition were driven by the more severe dementia diagnoses. The DAT group was split into two sub-groups based on a median split of their ACE-R scores. These two severity groups were then compared (using Mann-Whitney U-tests) to the control group on TASIT scores. The less severe DAT group had significantly lower median TASIT scores $($ TASIT-ERT median $=18$, TASITSIM median $=38$ and TASIT-SIE median $=34$ ) than the partner group (TASIT-ERT median $=24$, TASIT-SIM median $=49$ and TASIT-SIE median $=55$ ), all $p$ 's $<.001$. Similarly, the more severe group also had significantly lower median TASIT scores (TASIT-ERT median $=12.5$, TASIT-SIM median $=33$ and TASIT-SIE median $=33$ ) than the partner group, all $p$ 's $<.001$.

\section{Association Between Social Cognition and Cognitive Ability in Participants with DAT}

Correlations between social cognition scores and cognitive scores are presented in Table 3. TASIT total scores were not related to ACE-R total or to

\section{TABLE 3}

Spearman Correlations Between the ACE-R and TASIT ERT, SIM and SIE Scores

\begin{tabular}{|c|c|c|c|c|}
\hline & & $\begin{array}{c}\text { ACE-R } \\
\text { total }\end{array}$ & $\begin{array}{c}\text { BRCM } \\
\text { total }\end{array}$ & $\begin{array}{l}\text { NPI } \\
\text { total }\end{array}$ \\
\hline TASIT-ERT total & Rho & $0.57^{* *}$ & 0.03 & -0.16 \\
\hline ERT positive & Rho & $0.60^{* *}$ & 0.09 & -0.32 \\
\hline ERT negative & Rho & $0.53^{* *}$ & -0.00 & -0.08 \\
\hline TASIT-SIM total & Rho & 0.30 & 0.19 & -0.14 \\
\hline SIM sincere & Rho & 0.04 & 0.16 & -0.20 \\
\hline $\begin{array}{c}\text { SIM simple } \\
\text { sarcasm }\end{array}$ & Rho & 0.13 & 0.21 & -0.07 \\
\hline $\begin{array}{l}\text { SIM complex } \\
\text { sarcasm }\end{array}$ & Rho & $0.60 * *$ & 0.16 & -0.22 \\
\hline TASIT-SIE total & Rho & 0.30 & 0.04 & -0.13 \\
\hline SIE lie & Rho & -0.07 & 0.21 & -0.20 \\
\hline SIE sarcasm & Rho & 0.34 & 0.02 & 0.04 \\
\hline
\end{tabular}

Note. ACE-R: Addenbrooke's Cognitive Examination Revised; ERT: Emotion Recognition Test from TASIT, SIM: Social Inference-Minimal Test from TASIT SIE: Social Inference-Enriched Test from TASIT, Rho: Spearman's Correlation Coefficient, Sig.: Significance level.

** Significant at $p<.001$

any ACE-R sub scores. TASIT-ERT total scores were associated with ACE-R language ( $r h o=.55$, $p<.001)$ and ACE-R total ( $r h o=.57, p<.001)$ scores. Furthermore, scores for recognising both positive $(r h o=.60, p<.001)$ and negative $(r h o=$ $.53, p<.001)$ emotions on TASIT-ERT were also associated with ACE-R total scores. The TASITSIM overall score was not related to any ACE-R scores. However, the TASIT-SIM score for complex sarcasm items was related to fluency ( $r h o=$ 
TABLE 4

Neuro-Behavioural Problems in Participants with DAT Reported by Carers on the Neuropsychiatric Inventory

\begin{tabular}{llllll}
\hline & $N$ & Frequency & Severity & Total (Frequency $\times$ \\
Severity) & Distress \\
\hline Apathy & 20 & 50 & 27 & 75 & 43 \\
Anxiety & 14 & 29 & 19 & 47 & 27 \\
Irritability & 11 & 20 & 15 & 43 & 27 \\
Agitation & 10 & 13 & 12 & 20 & 18 \\
Appetite disturbances & 10 & 32 & 19 & 41 & 20 \\
Delusions & 4 & 7 & 4 & 8 & 9 \\
Hallucinations & 4 & 8 & 6 & 8 & 8 \\
Depression & 9 & 18 & 15 & 28 & 25 \\
Elation & 4 & 15 & 10 & 24 & 14 \\
Aberrant motor Behaviour & 8 & 18 & 6 & 13 & 13 \\
Disinhibition & 4 & 10 & 14 & 26 & 9 \\
Night time Behaviours & 6 & 11 & & 15 \\
\hline
\end{tabular}

$.56, p<.001)$, language $(r h o=.59, p<.001)$, visuospatial $(r h o=.58, p<.001)$ and total ACE-R $(r h o=.60, p<.001)$ scores. Neither the TASITSIE total score nor any of the SIE sub scores were related to any ACE-R scores.

Five participants with DAT who were unable to complete some or all of the sections in the TASIT also showed low-scores on the ACE-R total score. A comparison was made between the ACE-R scores of participants who had been able to complete all subtests on the TASIT and those who had not revealed significant differences in their ACE-R total scores $U(27)=107, Z=3.25, p<.001$.

\section{Neuro-Behavioural Results}

The NPI total scores were examined for participants with DAT. Apathy was reported by the most partners $(N=20)$, followed by anxiety $(N=14)$, irritability $(N=11)$, agitation $(N=10)$ and appetite changes $(N=10)$. Behaviours reported to occur most frequently were apathy, appetite disturbances, anxiety and irritability. Behaviours reported to be the most severe were apathy, anxiety, irritability and depression. Finally, behaviours reported to cause the most distress were apathy, anxiety, irritability and depression. Table 4 details the frequency, severity and distress scores for each behaviour assessed (Figure 1).

\section{Association Between Social Cognition and Behaviour Change in Participants with DAT}

Spearman correlations were conducted between social cognition scores (TASIT-ERT, SIM and SIE) and behaviour ratings (NPI total and NPI apa- thy, anxiety, depression, irritability and agitation). These behaviours were chosen for this analysis, given they were the most frequently encountered in our sample and most distressing as rated by partners. There were no significant correlations between social cognition scores and total behaviour ratings (all $p$ 's $<.05$ ). Similarly, no significant correlations were found between social cognition scores and distress levels for each of the behaviours or the total distress level as measured by the NPI.

\section{Association Between Social Cognition/Behaviour in Participants with DAT and Partner's Ratings of Relationship Continuity}

Spearman correlations revealed no significant correlations between partners' BRCM ratings and social cognition of participants with DAT as measured by TASIT-ERT, SIM and SIE (all $p$ 's <.05).

Significant negative correlations were found between the BRCM total scores and the NPI total scores, $r h o=-.70, p<.001$, and NPI total distress scores, rho $=-.71, p<.001$, suggesting that greater presence of problem behaviours and the distress they cause is related to poorer perceived continuity of the relationship. BRCM scores were significantly correlated with some specific behaviours on the NPI: 'apathy' (rho $=-.64, p<$ $.001)$, it's severity (rho $=-.67, p<.001)$ and how distressing it is for partners $(r h o=-.63$, $p<.001$ ), 'disinhibition frequency' (rho $=-.53$, $p<.001)$, severity $(r h o=-.53, p<.001)$ and how distressing it is for partners $(r h o=-.54, p<$ $.001)$ and 'agitation' ( $r h o=-.51 p<.001)$. None 


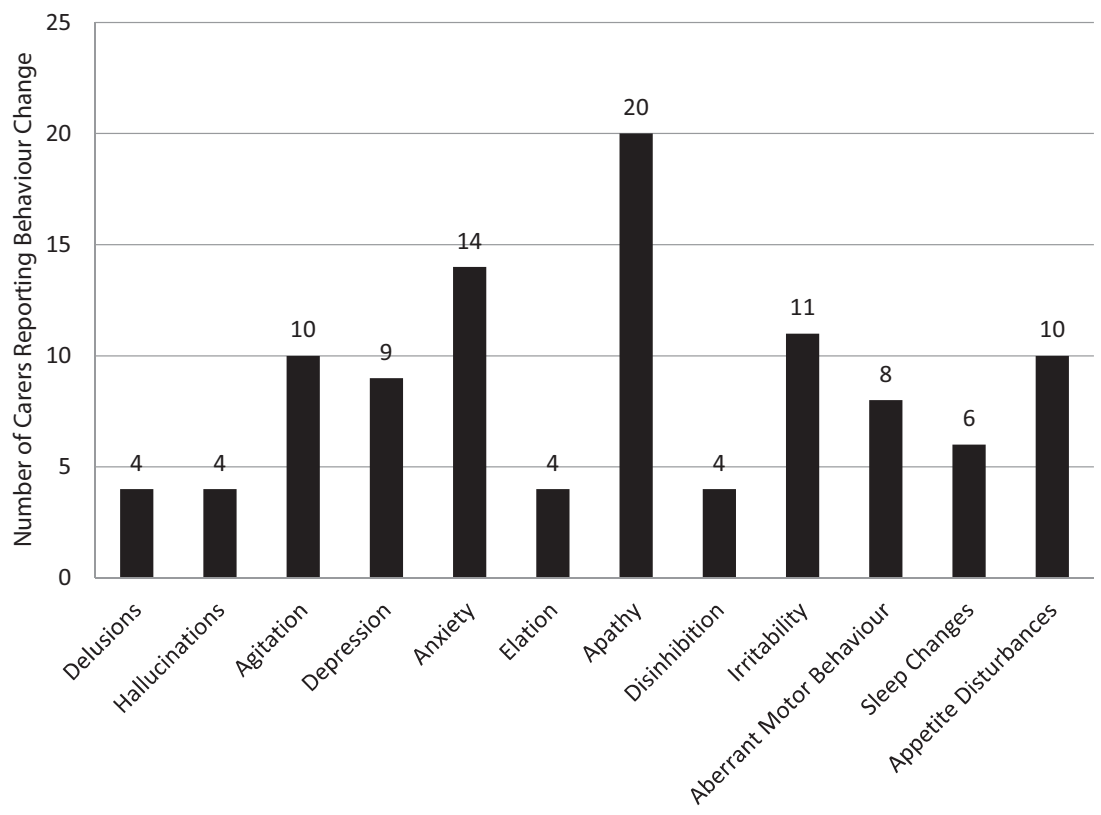

\section{FIGURE 1}

Frequency of behaviours as reported by carers in the NPI. Note. NPI: Neuropsychiatric Inventory.

of the other NPI behaviours were correlated with BRCM scores.

Because self-reported relationship continuity was related to the mood of the partner (HADS $\mathrm{A}: r h o=-.580, p=.001$ and HADS D: $r h o=$ $-.43, p=.022$ ), a second analysis strategy, a hierarchical linear regression, was used to determine whether NPI total scores could improve the prediction of self-reported relationship continuity over and above mood (measured by the HADS). This analysis was appropriate for these variables since errors on the BRCM were approximately normally distributed. The full model of HADS A, HADS D and NPI total score to predict BRCM score was statistically significant, $R^{2}=.632, F(2,27)=13.73$, $p<.0005$; adjusted $R^{2}=.886$. The addition of NPI score to the prediction of BRCM score from the HADS scores led to a statistically significant increase in $R^{2}$ of $.241, F(2,27)=15.71, p=.001$.

\section{Discussion}

This study examined impairments in social cognition in patients with DAT and whether these impairments were associated with behaviour changes. Furthermore, this study sought to determine whether these behaviour changes and social cogni- tion impairments were related to partner's perceptions of relationship continuity.

Participants with mild to moderate DAT showed difficulties on all three parts of TASIT compared to their partners' performance, demonstrating impaired social cognition. This is in line with previous research by Kipps et al. (2009b), using the TASIT-ERT, which suggested that people with DAT are poorer than controls at recognising emotions. Interestingly, this was true for both DAT patients with ACE-R scores higher than the median and for those with ACE-R score lower than the median, indicating that the social cognition impairment was not driven by a particularly impaired sub-group of participants with DAT. This suggests that social cognition impairments may be present even in the earlier stages of the disease when cognitive decline is mild. Despite this, general cognitive deficits did account for some of the variance in social cognition impairments, particularly with regard to emotion perception. These findings are in line with suggestions by Shany-Ur et al. (2012) that general cognitive decline may be partly responsible for difficulties in social cognition in participants with DAT. Interestingly though, parts 2 and 3 of the TASIT, the more complex parts, were largely unrelated to general cognition (with the exception of TASIT-SIM complex sarcasm). Thus, it 
seems that people with DAT are not only impaired at emotion recognition, but may also be impaired at decoding complex social messages, and that these impairments are not accounted for by general cognitive decline.

Phillips et al. (2010) reported that in order to successfully recognise emotions, individuals needed to rapidly detect the perceptual emotional stimulus and apply higher level decision making about what verbal descriptor best described a facial expression. It is thus plausible that emotion recognition skills require intact executive functioning skills, such as higher order decision-making skills (Phillips et al., 2010). There is evidence of a link between perceptual decline and general cognitive ability in DAT (Buck \& Radford, 2004). Indeed, on tasks with relatively low cognitive and perceptual requirements, participants with DAT were capable of recognising different emotions from nonverbal sources, including facial expressions (Bucks \& Radford, 2004; Burnham \& Hogervorst, 2004) and vocal prosody (Bucks \& Radford, 2004). However, most studies on social cognition have opted for a less sensitive (Feher et al., 1992), shorter, general cognitive ability test, the MMSE (Folstein, Folstein \& McHugh, 1975) (e.g., Phillips et al., 2010). The MMSE has shown significant sensitivity problems, particularly to frontal, linguistic and early memory difficulties (Feher et al., 1992). As such, results from studies using this measure as a predictor of social cognition should be interpreted with caution, as this scale may not fully capture impairments in executive and working memory functioning, which form a part of an individual's general cognitive skills. Thus, the current finding of an association between the ACE-R and social cognition provides evidence that social cognition impairments in DAT are at least partly attributable to general cognitive impairment.

The current study found impairments in participants with DAT on recognition of all emotions except for happy, adding to a growing body of evidence that recognition of positive emotions is preserved following DAT (Adolphs, 2001). This dichotomy is accompanied by evidence of distinct neural substrates for positive vs. negative emotion perception and processing within the brain (Adolphs, 2001). Lesion studies appear to show the amygdala is essential in the recognition of emotions from facial expressions, particularly negatively valanced emotions such as fear (Adolphs, 2001). Developmentally, the amygdala has played a crucial role in human survival, by providing almost automatic responses to negative emotions. On the other hand, Rosen et al. (2004) have indicated an association between the recognition of positive emotions and damage to the frontal re- gions of the brain, particularly in the behavioural variant of FTD. However, Rosenberg, McDonald, Dethier, Kessels, \& Westbrook (2014) explored the hypothesis of distinct neural pathways for positive and negative emotions in people with moderatesever traumatic brain injury. There findings suggested that rather than distinct pathways existing, happiness may simply be an 'easier' emotion to recognise as it relies on a single facial feature, whereas other emotions require additional information about the complex configuration of the face. They showed that the existing dichotomy in recognising positive and negative emotions may be better explained by a valence difficulty effect on the commonly used emotional expressions, further highlighting general affect recognition impairment (Rosenberg et al., 2014).

This study also sought to determine whether behaviour changes after DAT were underpinned by impairments in social cognition. This link was proposed in view that as disease progresses in dementia, social cognition may become impaired and this may lead to behaviour changes in the individual with DAT, due to difficulties understanding and responding to social interactions and feedback. No significant associations were found between any of the social cognition subtests and behaviour ratings in the NPI. These results are in contrast with Shimokawa et al. (2001) who found a relationship between interpersonal behaviour, including indifference to interpersonal relationships and difficulties with patient treatment/management, and recognition of static facial displays of emotion in a sample of 100 patients with a diagnosis of DAT. Studies with FTD patients have also established this relationship. For instance, Gregory et al. (2002) found an association between second-order ToM tasks and neuro-behavioural symptoms on the NPI. This inconsistency in the literature is likely driven by the array of different measurement tools used to tap social cognition and neuro-behavioural outcome.

Finally, this study aimed to determine whether behaviour changes and social cognition were predictive of partner's perceptions of the continuity of relationship. Correlational analyses revealed that social cognition was not related to partner's perceptions of relationship continuity. Adolphs (2001) theorised a close association between social cognition and functional outcomes on the basis that the ability to process social stimuli is essential for social interactions that are, in turn, essential for the continuation of functioning interpersonal relationships. Although there is evidence of this association between social cognition and interpersonal factors in other neurological patient groups (Bornhofen \& McDonald, 2008), no study up to now has 
assessed this relationship in neuro-degenerative conditions. It is also possible that participants with DAT compensate for social cognition difficulties in real-life situations by relying on other social and contextual cues and, thus, that it may not be important in predicting relationship quality. During conversation with couples, it became apparent that most partners' agreed that participants with DAT needed additional support in social situations to understand sarcasm or others' intentions. However, partners did not report this as significantly distressing. Qualitative observations of participants with DAT behaviour in social situations may be useful in understanding the extent of social cognition difficulties in DAT and the ways in which participants and families may have learnt to compensate for those impairments in real-life situations. Most of the evidence relating to partners' or carers' mood in neuro-degenerative conditions or ABI has focused on the effects of behaviour difficulties on carer burden or carers' mood, symptomatology where increased behaviour change has a significantly negative effect on carers' mood. Despite the lack of association found in the current study, a further exploration both qualitatively and quantitatively of this relationship may provide further insight into the needs and behaviour of individuals with a neuro-degenerative condition and the best support for their carers or partners.

Despite no association between social cognition and relationship continuity, the current study found that the presence, frequency and severity of behaviour changes in participants with DAT (particularly apathy, agitation or disinhibition) were negatively associated with relationship continuity. Furthermore, behavioural problems measured by the NPI total scores predicted relationship continuity even after accounting for the mood of the partners reporting. This result demonstrates that these behavioural changes are particularly debilitating and threaten the quality of the affected person's relationship with their intimate partner, who also is responsible for their care. Similarly, NPI scores have also been associated with a greater change in interpersonal relationships in braininjured populations (Osborne-Crowley, McDonald, \& Francis, 2016). These findings are particularly important when thinking about supporting an individual following a diagnosis of a possible dementia. In many services, the emphasis is placed on supporting the participants with DAT by providing strategies, medication, psycho-education or social interaction, and support for the partner, carer or family is usually only addressed as a reactive strategy or if problems arise and a possible breakdown in relationship is likely. Acknowledging the links between behaviour changes in DAT or mixed dementia and relationship continuity may help services prevent such breakdown and crisis situations by providing guidance and support for families and carers and diminish caregiving burden $(\mathrm{DoH}$, 2009). Placing such emphasis in supporting carers and families would also be in line with current national priorities, Scotland (DoH, 2009). This study has added to our understanding of the negative effects that behavioural changes in DAT can have on a couple's relationship quality. It has highlighted the importance of managing the behaviour changes in DAT, particularly 'disinhibition', 'apathy' and 'agitation', given the consequences it can have on relationship quality, and stresses the need to conduct more research into how to manage these behaviours in order to improve relationship satisfaction in a couple where one member has a diagnosis of DAT.

A few limitations of the current research should be noted. Foremost, the current study was unable to compare social cognition to another dementia patient group in order to determine whether they are a non-specific effect of pathology or a specific DAT effect. Furthermore, only TASIT was used to assess social cognition in this study. Considering the lack of a normative sample for older people on this task, it would have been beneficial to include additional ToM and emotion processing tasks in order to establish concurrent reliability and be able to determine 'impaired' performance and cut-off scores by examining standardised scores allowing for more detailed statistical methodology.

The current study was the first to assess the relationship between social cognition, behaviour and relationship continuity in DAT. Previous publications have commented on the lack of research available on the relationship between these factors (e.g., Kipps et al., 2009, Shany-Ur et al., 2012). Significant differences were found in the ability of people with DAT to correctly identify emotions and sarcasm, compared to their partners. Although social cognition scores did not appear to associate with behaviour changes in DAT, the present study found associations between behaviours such as apathy, disinhibition and agitation in DAT with relationship quality, supporting the need to focus interventions in management of these behaviours and minimise the impact on a couple's relationship status.

\section{Acknowledgements}

This research was conducted as part of the Doctorate in Clinical Psychology at the University of Edinburgh. This work was supported by the Scottish Dementia Clinical Research Network, who received funding from Scottish Ministers through the Chief Scientist Office. The views expressed in this 
publication are those of the authors and not necessarily those of Scottish Ministers or the Chief Scientist Office.

\section{Financial Support}

This research received no specific grant from any funding agency, commercial or not-for-profit sectors.

\section{Conflict of Interest}

None.

\section{Ethical Standards}

The authors assert that all procedures contributing to this work comply with the ethical standards of the relevant national and institutional committees on human experimentation and with the Helsinki Declaration of 1975, as revised in 2008.

\section{References}

Adolphs, R. (2001). The neurobiology of social cognition. Current Opinions in Neurobiology, 11(2), 11231-239.

Alzheimer's Society. (2012). Optimising treatment and care for people with behavioural and psychological symptoms of dementia. A best practice guide for health and social care professionals. Alzheimer's Society, UK.

American Psychiatric Association. (2013). Diagnostic and statistical manual of mental disorders (5th ed.). Arlington, VA: American Psychiatric Publishing.

Bornhofen, C. \& McDonald, S. (2008). Treating deficits in emotion perception following traumatic brain injury. Neuropsychological Rehabilitation, 18, 22-44

Bozeat, S., Gregory, C.A., Ralph, M.A., \& Hodges, J.R. (2000). Which neuropsychiatric and behavioural features distinguish frontal and temporal variants of frontotemporal dementia from Alzheimer's disease?. Journal of Neurology, Neurosurgery Psychiatry, 69, 178-86.

Bucks, R.S. \& Radford, S.A. (2004). Emotion processing in Alzheimer's disease. Aging and Mental Health, 8, 222-232.

Burke, J.R., \& Morgenlander, J.C. (1999). Managing common behavioral problems in dementia: How to improve quality of life for patients and families. Postgraduate Medicine, 106, 131-134.

Burnham, H., \& Hogervorst, E. (2004). Recognition of facial expressions of emotion by patients with dementia of the Alzheimer type. Dementia and Geriatric Cognitive Disorders, 18, 75-9.

Chow, T.W., Binns, M.A., Cummings, J.L., Lam, I., Black, S.E., Miller, B.L., . . v van Reekum, R. (2009). Apathy symptom profile and behavioural associations in frontotemporal dementia vs. dementia of
Alzheimer type. Archives of Neurology, 66, 888893.

Coen, R.F., Swanwick, G. R., O’Boyle, C. A, \& Coakley, D. (1997). Behaviour disturbance and other predictors of carer burden in Alzheimer's disease. International Journal of Geriatric Psychiatry, 12, 331336.

Cohen, C.A., Gold, D.P., Shulman, K.I., Wortley, J.T., McDonald, G., \& Wargon, M. (1993). Factors determining the decision to institutionalize dementing individuals: A prospective study. Gerontologist, 33, 714-720.

Cummings, J., Mega, M., Gray, K., RosenbergThompson, S., Carusi, D.A., \& Gornbein, J. (1994). The neuropsychiatric inventory: Comprehensive assessment of psychopathology in dementia. Neurology, 44, 2308-2314.

Cummings, J.L. (1997). The neuropsychiatric inventory: Assessing psychopathology in dementia patients. Neurology, 48(5 Suppl. 6), S10-S16.

Department of Health. (2009). Living well with dementia: A national dementia strategy. UK Government. London, UK.

Duff, K., Chelune, G.J., \& Dennett, (2011). Predicting estimates of premorbid memory functioning: Validation in a dementia sample. Archives of Clinical Neuropsychology, 26(6), 701-705

Ekman, P., \& Friesen, W.V. (1976). Pictures of facial affect. Palo Alto, CA: Consulting Psychological Press.

Feher, E.P., Mahurin, R.K., Doody, R.S., Cooke, N., Sims, J., \& Pirozzolo, F.J. (1992). Establishing the limits of the mini-mental state examination of 'subtests'. Archives of Neurology, 49, 87-92.

Field, A. (2009). Discovering statistics using SPSS. UK: SAGE.

Finkel, S.I., Costa e Silva, J., Cohen, G., Miller, S., \& Sartorius, N. (1996). Behavioral and psychological signs and symptoms of dementia: A consensus statement on current knowledge and implications for research and treatment. International Psychogeriatric, 8, 497-500.

Folstein, M.F., Folstein, S.E., \& McHugh, P.R. (1975). Mini-mental state: A practical method for grading the cognitive state of patients for the clinician. Journal of Psychiatric Research, 12(3), 189-98.

Gallagher-Thompson, D., Dal Canto, P., Jacob, T., \& Thomson, L. (2001). A comparison of marital interactions patterns between couples in which the husband does or does not have Alzheimer's disease. Journal of Gerontology, 56B(3), S140-S150.

Garand, L., Dew, M.A., Urda, B., Lingler, J.H., Dekosky, S.T., \& Reynolds, C.F. (2007). Marital quality in the context of mild cognitive impairment. Western Journal of Nursing Research, 29, 976-992.

Gregory, C., Lough, S., Stone, V., Erzinclioglu, S., Martin, L., Baron-cohen, S., \& Hodges, J.R. (2002). Theory of mind in patients with frontal variant frontotemporal dementia and Alzheimer's disease: Theoretical and practical implications, Brain, 125(4), $752-764$. 
Hargrave, R., Maddock, R. J., \& Stone, V. (2002). Impaired recognition of facial expressions of emotion in Alzheimer's disease. Journal of Neuropsychiatry and Clinical Neurosciences, 14, 64-71.

Hébert, R., Dubois, M.F., Wolfson, C., Chambers, L., \& Cohen, C. (2001). Factors associated with long-term institutionalization of older people with dementia: Data from the Canadian study of health and aging. Journal of Gerontoly, 56(11), 693-699.

Henry, J.D., Ruffman, T., McDonald, S., O’Leary, M.A.P., Phillips, L.H., Brodaty, H., \& Rendell, P.G. (2008). Recognition of disgust is selectively preserved in Alzheimer's disease. Neuropsychologia, 46(5), 1363-70.

Hodges, J.R., Patterson, K., Garrard, P., Bak, T., Perry, R., \& Gregory, C. (1999). The differentiation of semantic dementia and frontal lobe dementia (temporal and frontal variants of frontotemporal dementia) from early Alzheimer's disease: A comparative neuropsychological study. Neuropsychology, 13, 31-40.

Honan, C.A., McDonald, S., Sufani, C., Hine, D.W., \& Kumfor, F. (2016). The awareness of social inference test: Development of a shortened version for use in adults with acquired brain injury. Clinical Neuropsychologist, 30(2), 1-22.

Hsieh, S., Irish, M., Daveson, N., Hodges, J. R., \& Piguet, O. (2013). When one loses empathy: its effect on carers of patients with dementia. Journal of Geriatric Psychiatry and Neurology, 26(3), pp.174-184.

Jalbert, J.J., Daeillo, L.A., \& Lapane, K.L. (2008). Dementia of the Alzheimer type. Epidemiological Reviews, 30(1), 15-34.

Kaufer, D. I., Cummings, J. L., Christine, D., Bray, T., Castellon, S., Masterman, D., . . . DeKosky, S. T. (1998). Assessing the impact of neuropsychiatric symptoms in Alzheimer's disease: The neuropsychiatric inventory caregiver distress scale. Journal of the American Geriatric Society, 46, 210-215.

Keane, J., Calder, A.J., Hodges, J.R., \& Young, A.W. (2002). Face and emotion processing in frontal variant frontotemporal dementia. Neuropsychologia, 40 , 655-665.

Kipps, C.M., Nestor, P.J., Acosta-Cabronero, J., Arnold, R., \& Hodges, J.R. (2009b). Understanding social dysfunction in bvFTD: The role of emotion and sarcasm processing. Brain, 132(3), 592-603.

Kipps, M., Mioshi, E., \& Hodges, J.R. (2009a). Emotion, social functioning and activities of daily living in frontotemporal dementia. Neurocase, 15(3), 182189.

Koff, E., Zaitchik, D., Montepare, J., \& Albert, M.S. (1999). Emotion processing in the visual and auditory domains by patients with Alzheimer's disease. Journal of the International Neuropsychology Society, 5(1), 32-40.

Kumfor, F., Irish, M., Leyton, C., Miller, L., Lah, S., Devenney, E., Hodges, J.R. \& Piguet, O. (2015). Tracking progression of social cognition in neurodegenerative disorders. Journal of Neurology, Neurosurgery and Psychiatry, 85, 1076-1083.
Lavenu, I., Pasquier, F., Lebert, F., Petit, H., \& Van der Linden, M. (1999). Perception of emotion in frontotemporal dementia and Alzheimer disease. Alzheimer Disease and Associated Disorders, 13(2), 96-101.

McDonald, S., Bornhofen, C., Shum, D., Long, E., Saunders, C., \& Neulinger, K. (2006). Reliability and validity of The Awareness of Social Inference Test (TASIT): A clinical test of social perception. Disability and Rehabilitation, 28(24), 1529-1542.

McDonald, S., Flanagan, S., \& Rollins, J. (2002). The Awareness of Social Inference Test (TASIT). Pearson Assessment. London. UK.

McDonald, S., Flanagan, S., Rollins, J., \& Kinch, J. (2003). TASIT: A new clinical tool for assessing social perception after traumatic brain injury. Journal of Head Trauma Rehabilitation, 18, 219-238.

McKhann, G., Drachman, D., Folstein, M., Katzman, R., \& Price, D. (1984). Clinical diagnosis of Alzheimer's disease: Report of the NINCDS-ADRDA Work Group* under the auspices of Department of Health and Human Services Task Force on Alzheimer's Disease. Neurology, 34(7), pp. 939-944.

McKhann, G.M., Knopman, D.S., Chertkow, H., Hyman, B.T., Jack, C.R., Kawas, C.H., ... \& Mohs, R.C. (2011). The diagnosis of dementia due to Alzheimer's disease: Recommendations from the national institute on aging-Alzheimer's association workgroups on diagnostic guidelines for Alzheimer's disease. Alzheimer's \& Dementia, 7(3), 263-269.

Milders, M., Fuchs, S. \& Crawford, J.R. (2003). Neuropsychological impairments and changes in emotional and social behavior following severe traumatic brain injury. Journal of Clinical and Experimental Neuropsychology, 25, 157-172.

Milders, M., Ietswaart, M., Crawford, J.R., \& Currie, D. (2006). Social behavior following traumatic brain injury and its association with emotion recognition, understanding of intentions, and cognitive flexibility. Journal of the International Neuropsychological Society 14, 318-326.

Mioshi, E., Dawson, K., Mitchell, J., Arnold, R., \& Hodges, J.R. (2006). The Addenbrooke's Cognitive Examination Revised (ACE-R): A brief cognitive test battery for dementia screening. International Journal of Geriatric Psychiatry, 21(11), 10781085.

Morris, J. C. (1997). Clinical dementia rating: a reliable and valid diagnostic and staging measure for dementia of the Alzheimer type. International Psychogeriatrics. 9(1), pp. 173-176.

Morris, R.G., Worsley, C., \& Matthews, D. (2000). Neuropsychological assessment in older people: Old principles and new directions. Advances in Psychiatric Treatment, 6, 362-370.

O’Donnell, B. F., Drachman, D. A., Barned, H. J., Peterson, K. E., Swearer, J.M., \& Lew, R. A. (1992). Incontinence and troublesome behaviors predict 
institutionalisation in dementia. Journal of Geriatric Psychiatry and Neurology, 5, 45-52.

Osborne-Crowley, K., \& McDonald, S. (2016). Hyposmia, not emotion perception, is associated with psychological outcome after severe traumatic brain injury. Neuropsychology, EPub.https://doi.org/10. 1037/neu0000293.

Osborne-Crowley, K., McDonald, S. \& Francis, H. (2016). Development of an observational measure of social disinhibition after traumatic brain injury. Journal of Clinical and Experimental Neuropsychology, 38(3), 341-353.

Phillips, L.H., Scott, C., Henry, J.D., Mowat, D., \& Bell, J.S. (2010). Emotion perception in Alzheimer's disease and mood disorder in old age. Psychology and Aging, 25(1), 38-47.

Rankin, K.P., \& Salazar, A. (2009). Detecting sarcasm from paralinguistic cues: Anatomic and cognitive correlates in neurodegenerative disease. Neuroimage, 47(4), 2005-2015.

Rascovsky, K., Hodges, J., \& Knopman, D.S. (2011). Sensitivity of revised diagnostic criteria for the behavioural variant of frontotemporal dementia. Brain, 134, 2456-2477.

Riley, G.A., Fisher, G., Hagger, B.F., Elliott, A., Le Serve, H., \& Oyebode, J.R. (2013). The birmingham relationship continuity measure: The development and evaluation of a measure of the perceived continuity of spousal relationships in dementia. International Psychogeriatrics, 25, 263-274.

Roberts, V., Ingram, S., Lamar, M., \& Green, R. (1996). Prosody impairment and associated affective and behavioral disturbances in Alzheimer's disease. $\mathrm{Neu}$ rology, 47, 1482-1488.

Rosen, H.J., Narvaez, J.M., Hallam, B., Kramer, J.H., Wyss-Coray, C., Gearhart, R., Johnson, J.K., \& Miller, B.L. (2004). Neuropsychological and functional measures of severity in Alzheimer disease, frontotemporal dementia, and semantic dementia.
Alzheimer Disease Association and Related Disorders, 18, 202-207.

Rosenberg, H., McDonald, S., Dethier, M., Kessels, R.P.C., \& Westbrook, F.R. (2014). Facial emotion recognition following moderate-severe traumatic brain injury (TBI): Re-examining the valence effect and the role of emotion intensity. Journal of the International Neuropsychological Society, 20, 9941003.

Seeley, W.W., Crawford, R., Rascovsky, K., Kramer, J.H., Weiner, M., Miller, B.L., \& Gorno-Tempini, M.L. (2008). Frontal paralimbic network atrophy in very mild behavioral variant frontotemporal dementia. Archives of Neurology, 65, 249-255.

Shany-Ur, T., Poorzand, P., Grossman, S. N., Growdon, M. E., Jang, J. Y., Ketelle, R. S., Miller, B.L., \& Rankin, K. P. (2012). Comprehension of insincere communication in neurodegenerative disease: Lies, sarcasm, and theory of mind. Cortex; A Journal Devoted to the Study of the Nervous System and Behavior, 48(10), 1329-1341.

Shimokawa, A., Yatomi, N., Anamizu, S., Torii, S., Isono, H., Sugai, Y., \& Kohno, M. (2001). Influence of deteriorating ability of emotional comprehension on interpersonal behavior in Alzheimer-type dementia. Brain and Cognition, 47, 423-433.

Steele, C., Rovner, B., Chase, G. A, \& Folstein, M. (1990). Psychiatric symptoms and nursing home placement of patients with Alzheimer's disease. American Journal of Psychiatry, 147, 1049-1051.

The Psychological Corporation. (2001). Wechsler Test of Adult Reading, San Antonio, TX: Harcourt Assessment.

Van Hoesen, G.W., Parvizi, J., \& Chu, C.C. (2000). Orbitofrontal cortex pathology in Alzheimer's disease. Cerebral Cortex, 10, 1047-3211.

Zigmond, A.S., \& Snaith, R.P. (1983). The hospital anxiety and depression scale. Acta Psychiatrica Scandinava, 67, 361-70. 